\title{
Reward Processing in Children With Psychotic-Like Experiences
}

\author{
Jasmine Harju-Seppänen ${ }^{1,2, \oplus}$, Haritz Irizar ${ }^{2,3, \oplus}$, Elvira Bramon ${ }^{2, \oplus}$, Sarah-Jayne Blakemore ${ }^{4, \oplus}$, Liam Mason ${ }^{1,5,6,8, \oplus}$, and \\ Vaughan Bell ${ }^{*, 1,7,8, \oplus}$ \\ ${ }^{1}$ Research Department of Clinical, Educational and Health Psychology, University College London, London, UK; ${ }^{2}$ Division of \\ Psychiatry, University College London, London, UK; ${ }^{3}$ Genetics and Genomic Sciences, Icahn School of Medicine at Mount Sinai, \\ New York, NY, USA; ${ }^{4}$ Department of Psychology, University of Cambridge, Cambridge, UK; ${ }^{5}$ Max Planck University College London \\ Centre for Computational Psychiatry and Ageing Research, University College London, London, UK; ${ }^{6}$ Wellcome Trust Centre for \\ Human Neuroimaging, University College London, London, UK; ${ }^{7}$ South London and Maudsley NHS Foundation Trust, London, UK \\ ${ }^{8}$ Joint last authors. \\ *To whom correspondence should be addressed; UCL, Gower Street, London WC1E 6BT, UK; tel: +44 (0) 2076792000 , fax: +44 (0) 20 \\ 7679 2000, e-mail: Vaughan.Bell@ucl.ac.uk
}

Alterations to striatal reward pathways have been identified in individuals with psychosis. They are hypothesized to be a key mechanism that generate psychotic symptoms through the production of aberrant attribution of motivational salience and are proposed to result from accumulated childhood adversity and genetic risk, making the striatal system hyper-responsive to stress. However, few studies have examined whether children with psychotic-like experiences (PLEs) also exhibit these alterations, limiting our understanding of how differences in reward processing relate to hallucinations and delusional ideation in childhood. Consequently, we examined whether PLEs and PLE-related distress were associated with reward-related activation in the nucleus accumbens (NAcc). The sample consisted of children $(N=6718)$ from the Adolescent Brain Cognitive Development (ABCD) study aged 9-10 years who had participated in the Monetary Incentive Delay (MID) task in functional MRI. We used robust mixedeffects linear regression models to investigate the relationship between PLEs and NAcc activation during the reward anticipation and reward outcome stages of the MID task. Analyses were adjusted for gender, household income, ethnicity, depressive symptoms, movement in the scanner, pubertal development, scanner ID, subject and family ID. There was no reliable association between PLEs and alterations to anticipation- or outcome-related striatal reward processing. We discuss the implications for developmental models of psychosis and suggest a developmental delay model of how PLEs may arise at this stage of development.
Key words: psychotic-like experiences/childhood/fMRI

\section{Introduction}

Psychotic-like experiences (PLEs) include delusionlike beliefs and hallucinations that remain below the threshold for psychotic disorder. PLEs are relatively common in childhood ${ }^{1}$ with a median prevalence of $17 \%$ among children aged $9-12$ years and $7.5 \%$ among those aged $13-18$ years. ${ }^{2}$ Even during childhood, however, they are a predictor of later transition to psychosis $^{3-5}$ and poor physical and mental health outcomes across the lifespan..$^{2,4,6,7}$

It is unclear how childhood PLEs relate to established neurocognitive mechanisms for adult psychosis. One hypothesis is that both adult and childhood PLEs are associated with altered striatal dopamine. ${ }^{8}$ In the developmental risk factor model, dysregulated striatal dopamine is cited as a final common pathway where genetic risk and developmental adversity converge and lead to psychosis via the generation of aberrant salience ${ }^{9,10}$ - a process where typically innocuous experiences are assigned heighted motivational salience due to misfiring of striatal dopamine leading to delusions and perceptual aberrations. Indeed, neuroimaging studies on reward processing in psychosis risk states and prodromal periods indicate that dysregulation of striatal dopamine is detectable before the onset of frank psychotic disorder (reviewed in Howes et $\mathrm{al}^{11}$ ). Initial evidence suggests that there is an earlier association between dysregulated striatal reward processing and PLEs in 14-19-year-old adolescents. ${ }^{12}$ 
However, it is still not clear if altered striatal reward processing that predicts psychosis and psychosis-risk in older individuals would necessarily explain the presentation of PLEs at a younger age. This is important because childhood is a crucial point of risk divergence for PLEs. An estimated $75 \%-90 \%$ of psychotic experiences during childhood and adolescence are transitory ${ }^{13,14}$ but those whose PLEs do not resolve have particularly poor outcomes ${ }^{15,16}$ with distress related to PLEs at age 12 adding predictive value for poor outcome later in life. ${ }^{17}$ However, PLEs at ages 8-15 years show a weaker relationship with later poor outcome than PLEs at ages 16 and over, despite a greater prevalence at this earlier age, ${ }^{18}$ suggesting they may not fully reflect the same mechanism as PLEs in later adolescence. Consequently, understanding whether dysregulated reward processing is associated with PLEs and PLE-related-distress during earlier childhood could provide important evidence to understand to what extent these experiences reflect an early disruption to a key causal mechanism present in later psychosis. The aim of the current study was therefore to examine whether PLEs and PLE-related distress in childhood is associated with alterations to striatal reward processing.

Reward processing consists of both reward anticipation and reward evaluation and that these functions of the reward system dissociate. ${ }^{19}$ The monetary incentive delay (MID) task was designed to distinguish these functions when used in functional magnetic imaging (fMRI) studies. ${ }^{20}$ It has been used extensively in psychosis research and meta-analysis of relevant fMRI studies provide strong evidence for striatal reward system dysregulation in adults with frank psychosis. ${ }^{21}$ Additional studies have also found evidence for these alterations in antipsychotic naive patients with schizophrenia, ${ }^{22}$ and in adults with PLEs. ${ }^{23}$ Evidence from concurrent fMRI and positron emission tomography (PET) indicates that changes to dopamine transport underlie changes in fMRI reward system-related activation during the MID, ${ }^{24}$ suggesting that fMRI studies of the MID task are a reliable proxy for alterations to reward-related dopamine function. Studies with children indicate that the paradigm is valid for measuring reward processing in this age group. ${ }^{25}$

Small sample sizes and lack of representative sampling are a challenge for fMRI studies ${ }^{26}$ and this has been cited as a particular issue for neuroimaging studies of children. ${ }^{27}$ Here, we aimed to test whether PLEs or PLErelated distress was associated with dysregulated rewardprocessing in the left and right nucleus accumbens (NAcc) during childhood by examining the association between activation during the fMRI MID task in a large $(N=6900+)$ sample of 9-10-year-olds, who were part of the Adolescent Brain Cognitive Development (ABCD) study. ${ }^{28}$ The $\mathrm{ABCD}$ study is an ongoing cohort study including more than 11000 children and includes extensive social, cognitive, and developmental measures. It includes demographics, measures of PLEs and PLE-related distress and, in over half of children, the MID task in fMRI.

Consequently, we aimed to test whether PLEs or PLErelated distress could be explained by dysregulated striatal reward processing by examining the association between NAcc activation during the fMRI MID task in a large $(N=6500+)$ sample of 9-10-year-olds. We tested both anticipation- and outcome-related reward processing in the left and right NAcc while controlling for potential confounders.

\section{Methods}

\section{Sample}

The ABCD dataset (release 3.0; https://abcdstudy.org/) includes 11878 children aged $9-10$ years. ${ }^{29}$ This is a longitudinal dataset being collected at 21 sites across the US. Full details of recruitment are described in Garavan et al. ${ }^{28}$ Institutional review board approval was obtained for each site before data collection and all parents provided written informed consent in addition to assent from the participants. ${ }^{30}$

Data from participants was excluded based on the following criteria: having a psychiatric diagnosis $(N=1973)$, not completing the Prodromal Questionnaire $(N=12)$, taking psychotropic medication $(N=1032)$, not completing the MID task in the scanner $(N=1030)$, insufficient performance on the task $(N=573)$, missing motion data $(N=455)$, missing fMRI data $(N=27)$, and if reward-related activation in the NAcc was more than three standard deviations from the mean $(N=223$ for reward anticipation; $N=123$ for reward outcome), leaving $N=6718$ who contributed to either the final anticipation or outcome analysis.

\section{Measures}

Psychotic-Like Experiences. PLEs were measured using the Prodromal Questionnaire-Brief Child Version, a modified version of the Prodromal Questionnaire Brief Version (PQ-B) ${ }^{31}$ - a self-report measure for psychosis risk syndromes that has been validated in 9-10-yearolds. ${ }^{32}$ Unlike the Kiddie Schedule for Affective Disorders and Schizophrenia (KSADS-5), also available in the $\mathrm{ABCD}$ dataset, the PQ-BC allows for measurement of PLEs alongside a measure of distress for the same items. The PQ-BC is a 21-item questionnaire that measures unusual perceptions and sensations, ideas of reference, affective changes, unusual beliefs, or abnormally suspicious thoughts, along with associated distress. The PQ-BC consists of two parts: the first asks whether the individual has had any of the listed psychotic-like thoughts, feelings and experiences, with an overall score ranging from 0 to 21. If they answer yes, participants also indicate how related distressing in the second part (from 1 to 5). A subset of six items were selected to represent analogues 
of positive symptoms of psychosis (thought interference, visual hallucination, auditory hallucination, two items for paranoia and bizarre beliefs). PLE types were derived from this variable, where participants were categorized as having no PLEs, nondistressing PLEs, or distressing PLEs. Additional analyses used total sum of PLEs and PLE-related distress.

Depressive Symptomology. Depressive symptoms were measured using the Kiddie Schedule for Affective Disorders and Schizophrenia (KSADS-5). The ABCD study used a recently validated and computerized version of the KSADS-5..$^{33}$ The following depressive symptoms were added to create a depression score: depressed mood, anhedonia, and irritability.

Pubertal Development. Pubertal development was measured using the Pubertal Development Scale (PDS). ${ }^{34}$ Child-provided data was used as the primary measure of pubertal stage and missing scores were supplemented by parent-reported information.

Monetary Incentive Delay Task. MID task ${ }^{20}$ measures the anticipation and receipt of rewards and losses. Participants are presented with an incentive cue $(2000 \mathrm{~ms}$ ) at the beginning of each trial (Win $\$ 5$, Win $\$ 0.20$, Lose $\$ 0.20$, Lose $\$ 5$ or $\$ 0$-no money at stake), followed by a jittered anticipation event (lasting 1500-4000 ms). Participants then need to respond to a variable target (150-500 ms), to either win or avoid losing money. In the ABCD study, participants are presented with 40 rewards (20 small reward, 20 large reward) and 40 loss anticipation trials (20 small loss, 20 large loss), 20 no money anticipation trials, and feedback trials. ${ }^{35}$ The task was individualized with the initial duration of the response target drawn from a practice session completed by the participant prior to entering the scanner. In order to reach a $60 \%$ accuracy rate, task difficulty was adjusted during the task after every third incentivized trial based on the overall accuracy rate of the previous six trials. The target duration was shortened if the individual's accuracy fell below the target accuracy level. Participants who did not reach acceptable performance in the task were excluded from analysis (indexed by whether all trial types resulted in more than three events for both positive and negative feedback), as well as those whose NAcc activity was above or below three standard deviations. The MID task has been previously validated in typically developing children during fMRI ${ }^{36}$ and validation studies for the paradigm and data used in this study have been previous by Casey et $\mathrm{al}^{35}$ and Chaarani et al. ${ }^{37}$ Casey et $\mathrm{a}^{35}$ reported that the experimental manipulation was successful in maintaining hit rates at close to $60 \%$, and that reaction times and payoff amounts were consistent across experimental runs. Chaarani et $\mathrm{al}^{37}$ reported that the task is associated with robust brain activations which are consistent with the extant literature.

Imaging Acquisition. The primary outcome was rewardrelated activation during the MID task from the left and right NAcc. Full details on imaging acquisition is reported in Casey et al. ${ }^{35}$ Imaging data were collected across sites using multichannel coils and multiband echo planar imaging acquisition. Scanning included a fixed order of localizer, T1- and T2-weighted images, resting state, and diffusion-weighted imaging. Three tasks (MID task, stop signal, and emotional n-back) were completed in an order randomized across participants. Blood-oxygenlevel-dependent (BOLD) images were acquired using gradient EPI with standardized acquisition parameters.

Imaging Processing and Analysis. The ABCD Data Analysis and Informatics Center performed centralized processing and analysis of the imaging data. Full information regarding this is detailed in Hagler et al, ${ }^{38}$ and is summarized here. Left and right NAcc regions of interest were derived from subcortical segmentation using FreeSurfer 5.3.0. ${ }^{39}$ Estimated task-related activation were computed for individual subjects using the general linear model in AFNI 3dDeconvolve and were available as contrast beta weights. The contrasts used in this study were "large reward versus no money" and "small reward versus no money" for reward anticipation activity, and "all reward positive versus negative feedback" for reward outcome activity. For these contrasts, region of interest average beta coefficients were computed for each of the two runs and then averaged.

Statistical Analysis. We conducted analyses to investigate the association between PLEs, presence of any nondistressing PLEs, and presence of any distressing PLEs, and reward-related activation in the NAcc using multilevel regression analyses across the population sample. Each analysis was conducted and reported separately for two outcomes: left and right NAcc activation during the reward anticipation stage of the MID task, and left and right NAcc activation during reward outcome stage of the MID task. We tested for evidence of heteroscedasticity in the data, and due to its presence, estimated the effects of the predictor variables using robust mixed effects linear regression models. ${ }^{40}$

For all analyses, we initially tested for a minimally adjusted association between PLE type and rewardrelated activation, adjusted only for the random effects covariates (subject ID, nested within family ID, and scanner ID). We then subsequently updated the model to include additional fixed effect covariates to test the association after adjustment for potential confounders. These included sex, household income, parental education, ethnicity, motion in scanner, depressive symptoms, and pubertal development. 
Sex, household income, parental education, and ethnicity were included as potential confounders owing to their association with psychosis risk. ${ }^{41-43}$ Depression was included as a potential confounder due to its association with alterations in reward processing. ${ }^{44}$ Pubertal development was included as a potential confounder due to associations between reward processing and puberty. ${ }^{45}$ Motion in scanner was included due known role as a confounder in fMRI activation studies.

Missing data for the covariates was imputed through multiple imputation using the Mice package in R. ${ }^{46}$ Polytomous regression was used for unordered factor variables. Proportional odds model was used for ordered factor variables. Logistic regression imputation was used for binary variables.

We subsequently repeated the main analyses but included all individuals with psychiatric diagnoses and medication known to have significant impact on reward processing (stimulants and antipsychotics, see supplementary tables 1-4). We also completed alternative analysis where PLEs were included as sum total, along with their associated distress (see supplementary tables 5-8). Finally, we performed analyses only in individuals with a psychiatric diagnosis (see supplementary tables 9 and $10)$.

All analyses were conducted in $R$ (version 3.6.2) using the robustlmm package. ${ }^{40}$ The data were transformed into long format using reshape ${ }^{47}$ to allow us to test for multivariate outcomes. All analysis code and analysis output for this study has been made freely available on an Open Science Framework archive: https://osf. io/vqzhu/?view_only=3851605a5ab74267ab68b35c207 ef90a.

\section{Results}

After applying our exclusion criteria, 6718 participants remained for either the reward anticipation $(N=6553)$ and/or reward outcome $(N=6654)$ analyses. The demographic characteristics of the sample are shown in table 1 .

\section{Effect of PLEs and Distress on Reward Anticipation}

As can be seen from tables 2 and 3, there were main effects of reward magnitude and laterality on reward anticipation activity, indicating the validity of the paradigm, even after adjustment for potential confounders. However, there was no association with non-distressing or distressing PLEs in either analysis. Effects of PLE type on NAcc activation for reward anticipation are displayed by left and right laterality in figure 1 .

\section{Effect of PLEs and Distress on Reward Outcome}

As shown in tables 4 and 5, there were main effects of NAcc laterality on reward anticipation activity, even after adjustment for potential confounders, but no association with non-distressing or distressing PLEs in either analysis. Effects of PLE type on NAcc activation for reward outcome are displayed by left and right laterality in figure 2.

In addition, we completed sensitivity analyses reported in supplementary tables 1-4 that included all individuals, including those with a psychiatric diagnosis or medication use. The pattern of results was similar across analyses with regard to PLEs. The only exception was that PLE-related distress was significantly associated

Table 1. Demographic Characteristics of Participants Who Contributed to Either the Reward Anticipation or Outcome Analysis $(N=6718)$

\begin{tabular}{|c|c|c|c|c|}
\hline & $\begin{array}{c}\text { Total } \\
(N=6718)\end{array}$ & $\begin{array}{l}\text { No PLEs } \\
(N=4025)\end{array}$ & $\begin{array}{c}\text { Non-distressing } \\
\text { PLEs } \\
(N=833)\end{array}$ & $\begin{array}{l}\text { Distressing PLEs } \\
\quad(N=1860)\end{array}$ \\
\hline Age (SD) & $9.9(0.62)$ & $9.9(0.62)$ & $9.9(0.62)$ & $9.9(0.62)$ \\
\hline \multicolumn{5}{|l|}{ Gender, $N(\%)$} \\
\hline Female & $3494(52.0 \%)$ & $2107(52.3 \%)$ & $386(46.3 \%)$ & $1001(53.8 \%)$ \\
\hline \multicolumn{5}{|c|}{ Household income (USD) } \\
\hline$<50 \mathrm{~K}$ & $1872(27.9 \%)$ & $984(24.4 \%)$ & $236(28.3 \%)$ & $652(35.1 \%)$ \\
\hline$\geq 50 \mathrm{~K}$ and $<100 \mathrm{~K}$ & $1866(27.8 \%)$ & $1090(27.1 \%)$ & $237(28.4 \%)$ & $539(29.0 \%)$ \\
\hline$\geq 100 \mathrm{~K}$ & $2980(44.4 \%)$ & $1951(48.5 \%)$ & $360(43.2 \%)$ & $669(36.0 \%)$ \\
\hline \multicolumn{5}{|c|}{ Parental education, $N(\%)$} \\
\hline$<$ HS diploma & $398(5.9 \%)$ & $181(4.5 \%)$ & $49(5.9 \%)$ & $168(9.0 \%)$ \\
\hline HS diploma/GED & $641(9.5 \%)$ & $347(8.6 \%)$ & $76(9.1 \%)$ & $218(11.7 \%)$ \\
\hline Some college & $1847(27.5 \%)$ & $989(24.6 \%)$ & $262(31.5 \%)$ & $596(32.0 \%)$ \\
\hline Bachelor & $2002(29.8 \%)$ & $1276(31.7 \%)$ & $230(27.6 \%)$ & $496(26.7 \%)$ \\
\hline Postgraduate degree & $1830(27.2 \%)$ & $1232(30.6 \%)$ & $216(25.9 \%)$ & $382(20.5 \%)$ \\
\hline \multicolumn{5}{|l|}{ Ethnicity } \\
\hline Asian & $172(2.6 \%)$ & $114(2.8 \%)$ & $22(2.6 \%)$ & $36(1.9 \%)$ \\
\hline Black & $886(13.2 \%)$ & $437(10.9 \%)$ & $124(14.9 \%)$ & $325(17.5 \%)$ \\
\hline Other/mixed & $1193(17.8 \%)$ & $647(16.1 \%)$ & $166(19.9 \%)$ & $380(20.4 \%)$ \\
\hline White & $4467(66.5 \%)$ & $2827(70.2 \%)$ & $521(62.5 \%)$ & $1119(60.2 \%)$ \\
\hline
\end{tabular}

Page 4 of 10 
Table 2. Minimally Adjusted Regression Model $(N=6553)$ Examining the Effect of Presence and Type of PLEs, Reward Magnitude and Laterality on Nucleus Accumbens (NAcc) Response to Reward Anticipation

\begin{tabular}{lcc}
\hline Predictor & Estimate & $95 \%$ CIs \\
\hline Non-distressing PLEs & -0.001 & $-0.015,0.013$ \\
Distressing PLEs & -0.006 & $-0.017,0.004$ \\
Laterality (right NAcc $>$ left NAcc) & -0.012 & $-0.017,-0.008$ \\
Reward magnitude (large reward $>$ small reward) & 0.072 & $0.068,0.077$ \\
\hline
\end{tabular}

Table 3. Fully Adjusted Regression Model $(N=6553)$ Examining the Effect of Presence and Type of PLEs, Reward Magnitude and Laterality on NAcc Response to Reward Anticipation

\begin{tabular}{|c|c|c|c|}
\hline Predictor & Estimate & $95 \% \mathrm{CIs}$ & $P$ \\
\hline Non-distressing PLEs & 0.0005 & $-0.014,0.015$ & .949 \\
\hline Distressing PLEs & -0.002 & $-0.012,0.009$ & .755 \\
\hline Laterality (right NAcc > left NAcc) & -0.012 & $-0.017,-0.008$ & $<.001$ \\
\hline Reward magnitude (large reward > small reward) & 0.072 & $0.068,0.077$ & $<.001$ \\
\hline Gender & 0.001 & $-0.009,0.010$ & .912 \\
\hline Depressive symptoms & -0.005 & $-0.019,0.009$ & .461 \\
\hline Household income $[<50 \mathrm{~K}]$ & -0.001 & $-0.015,0.013$ & .856 \\
\hline Household income $[\geq 50 \mathrm{~K}$ and $<100 \mathrm{~K}]$ & -0.001 & $-0.013,0.010$ & .798 \\
\hline Parental education $-<$ HS diploma & -0.023 & $-0.046,-0.0001$ & .049 \\
\hline Parental education-HS diploma/GED & -0.025 & $-0.044,-0.007$ & .007 \\
\hline Parental education-Post graduate degree & -0.007 & $-0.019,0.005$ & .231 \\
\hline Parental education-Some college & 0.002 & $-0.015,0.011$ & .755 \\
\hline Race-Asian & -0.020 & $-0.049,0.008$ & .162 \\
\hline Race_Black & -0.003 & $-0.018,0.012$ & .698 \\
\hline Race-Other & -0.005 & $-0.017,0.008$ & .464 \\
\hline Motion & -0.036 & $-0.057,-0.016$ & $<.001$ \\
\hline Pubertal development & -0.005 & $-0.015,0.005$ & .338 \\
\hline
\end{tabular}

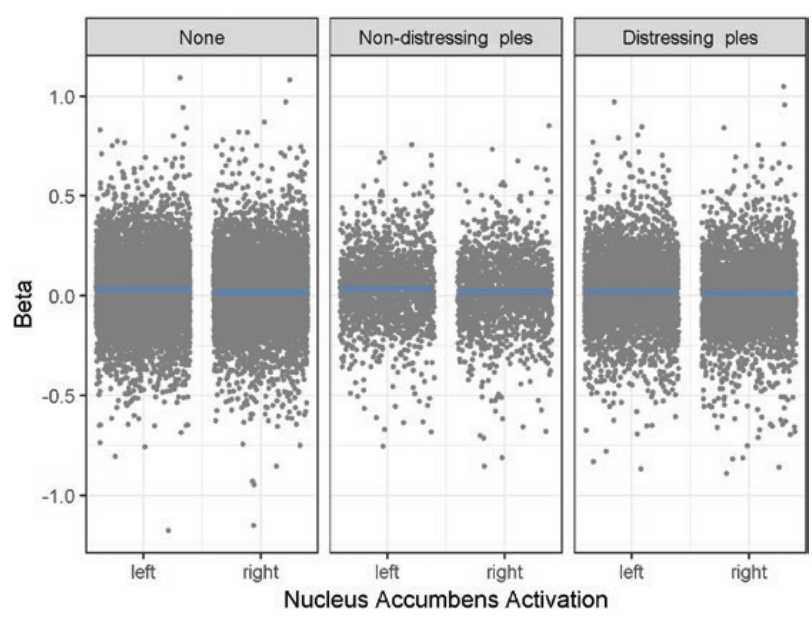

Fig. 1. Relationship between PLE group status on left and right nucleus accumbens (NAcc) activation in the reward-anticipation component of the Monetary Incentive Delay task.

with striatal activation in the minimally adjusted reward anticipation analysis $(\beta=-0.013,95 \% \mathrm{CI}=-0.022$, $-0.004, P=0.0055)$. However, this relationship became non-significant in the fully adjusted analysis $(\beta=-0.010$, $95 \% \mathrm{CI}=0.018,0.0001, P=.053)$.
We also completed additional analysis examining the effect of PLEs and PLE-related distress by coding them as sum total variables: total number of PLEs and total levels of PLE-related distress. Total number of PLEs was not related to anticipation-related reward activation $(\beta=<0.001,95 \% \mathrm{CI}=-0.006,0.007, P=0.883)$ or reward outcome-related reward activation $(\beta=0.001,95 \%$ $\mathrm{CI}=-0.007,0.009, P=.855)$ in minimally adjusted analyses. Similarly, total level of PLE-related distress was not related to anticipation-related reward activation $(\beta=-0.002,95 \% \mathrm{CI}=-0.005,0.0002, P=0.068)$ or reward outcome-related reward activation $(\beta=-0.001$, $95 \% \mathrm{CI}=-0.004,0.002, P=.460)$ in minimally adjusted analyses. This pattern of relationships remained unchanged in the fully adjusted analyses (full details are given in supplementary tables 5-8). We also performed additional analyses including only the subset of individuals with a psychiatric diagnosis (supplementary tables 9 and 10). These analyses indicated that there was no reliable association between PLEs and reward outcome. In this same subset of participants, distressing PLEs (but not non-distressing PLEs) were associated with reward anticipation activation only, showing an association with a small reduction in reward-anticipation-related activity $(\beta=-0.033,95 \% \mathrm{CI}=-0.055,-0.012, P=.003)$. 
Table 4. Minimally Adjusted Regression Model $(N=6654)$ on Association Between Types of PLEs, Distress, Laterality on NAcc Response to Reward Outcome

\begin{tabular}{|c|c|c|c|}
\hline Predictor & Estimate & $95 \%$ CIs & $P$ \\
\hline Non distressing PLEs & -0.004 & $-0.021,0.013$ & 0.670 \\
\hline Distressing PLEs & -0.006 & $-0.019,0.006$ & 0.320 \\
\hline Laterality (Right NAcc > Left NAcc) & -0.025 & $-0.030,-0.021$ & $<0.001$ \\
\hline
\end{tabular}

Table 5. Fully Adjusted Regression $\operatorname{Model}(N=6654)$ on Association Between Types of PLEs, Distress, Laterality on NAcc Response to Reward Outcome

\begin{tabular}{lcr}
\hline Predictor & Estimate & \multicolumn{1}{c}{$95 \%$ CIs } \\
\hline Non-distressing PLEs & -0.005 & $-0.022,0.012$ \\
Distressing PLEs & -0.008 & $-0.021,0.005$ \\
Laterality (right NAcc $>$ left NAcc) & -0.025 & $-0.030,-0.021$ \\
Gender & 0.008 & $-0.003,0.019$ \\
Depressive symptoms & 0.009 & $-0.007,0.026$ \\
Household income [<50K] & 0.002 & $-0.015,0.019$ \\
Household income [ $\geq 50 \mathrm{~K}$ and <100K] & -0.00002 & $-0.013,0.014$ \\
Parental education-<HS diploma & -0.009 & $-0.037,0.018$ \\
Parental education-HS diploma/GED & -0.006 & $-0.029,0.016$ \\
Parental education-Post graduate degree & -0.006 & $-0.021,0.008$ \\
Parental education-Some college & -0.002 & $-0.017,0.014$ \\
Race-Asian & 0.022 & $-0.013,0.058$ \\
Race-Black & -0.025 & $-0.044,-0.007$ \\
Race-Other & -0.012 & $-0.027,0.003$ \\
Motion & 0.075 & .812 \\
Pubertal development & 0.002 & .981 \\
\hline
\end{tabular}

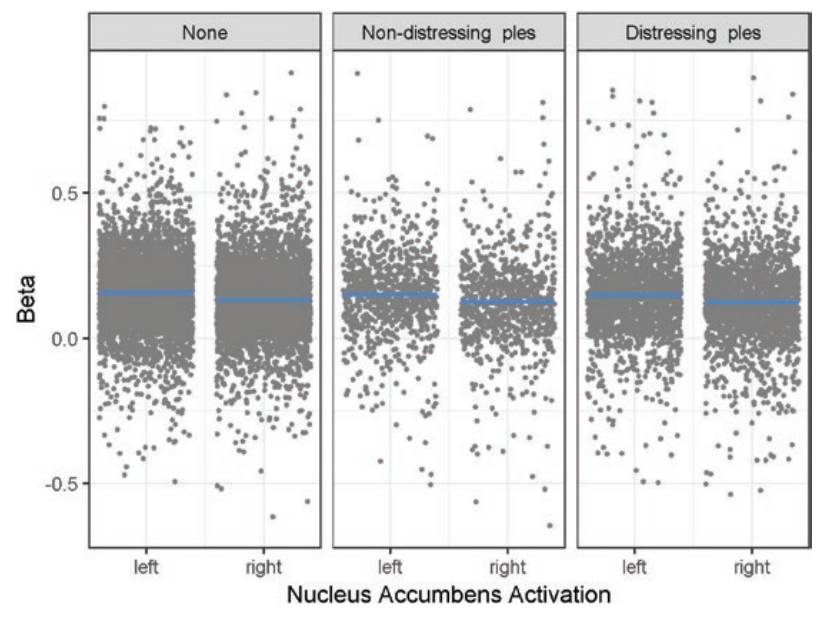

Fig. 2. Relationship between PLE group status on left and right NAcc activation in the reward-outcome component of the MID task.

\section{Discussion}

In this large study of over 6500 9-10-year-old children, we report no association between PLEs and NAcc activation during either the reward anticipation or reward outcome stages of an fMRI MID task. This was the case regardless of whether PLEs were included as a categorical or continuous variable. We completed a number of further supplementary analyses, including all individuals who were previously excluded due to the exclusion criteria, including PLE-related distress as a continuous measure, and including only participants with a psychiatric diagnosis. In these further analyses, only one statistical association was found: distressing PLEs (but not non-distressing PLEs) were associated with NAcc activation during reward anticipation (but not reward outcome) in the subpopulation of participants with psychiatric diagnoses. Given that this result was the only significant association from a large number of tests, was a small effect, was conducted as an exploratory analysis, and was not present for PLEs without distress in the same analysis, we suggest it is unlikely to be strong evidence for the presence of this mechanism.

The findings have several implications for developmental models of psychosis risk and our understanding of explanatory mechanisms for psychosis-spectrum experiences more broadly. In terms of the psychosis risk, the developmental risk factor mode ${ }^{10}$ suggests that accumulated childhood and social adversity combined with genetic risk makes the striatal dopamine system hyper-responsive to stress. According to the model, these alterations generate the symptoms of psychosis through a process of aberrant assignment of salience to stimuli that would normally appear to have low levels of motivational significance. ${ }^{9,48}$ These findings have been supported by fMRI studies reporting dysregulated reward processing in the MID task in adults with psychosis ${ }^{49,50}$ and in adolescents with PLEs. ${ }^{12}$ 
This study found no evidence for the presence of this mechanism at a peak age for PLEs in 9-10-year-old children despite a very large sample and validated measures that have produced reliable evidence for this association in older age groups. We also found no strong evidence for this when we included children with psychiatric diagnoses, who typically share a greater number of risk factors for reward system sensitization. This is despite the fact that PLEs in children of this age predict poor outcome over the lifespan, ${ }^{15,16}$ poorer cognitive abilities $^{51}$ and greater levels of adversity. ${ }^{52}$ This suggests that PLEs at this age are markers of adverse development and/or psychopathology but are potentially not associated with alterations to striatal activation, raising doubts over whether they share a mechanism proposed for psychotic-spectrum experiences later in life.

Notably, preadolescents have been shown to have several perceptual and reasoning differences that alter and stabilize during adolescence, potentially suggesting other mechanisms that might generate PLEs. For example, there is evidence that preadolescent children may perform auditory functions more unreliably than adults, tending to rely more heavily on top-down interpretation of sounds. ${ }^{53}$ Similarly, in the visual domain, preadolescent children tend to rely more on high spatial frequencies to extract local facial features to perceive fearful facial expressions whereas adolescent children use rapid decoding of global features using in the low spatial frequency ranges. ${ }^{54}$ Additionally and relevant to the measurement of unusual beliefs, magical thinking is common in childhood although declines into adolescence and this is largely understood in terms of the under-development of causal reasoning ${ }^{55}$ involving the understanding of transfer of physical force between objects, the outcomes of goal-directed actions produced by dispositional agents, and the ability to track covariation relations between events. Development in each of these domains may additionally be affected by developmental adversities, potentially giving rise to PLEs during preadolescence that are generated by distinct mechanisms from PLEs reported later in life.

Based on current findings, we hypothesize that PLEs in preadolescence may be generated by delayed development of perceptual and causal reasoning. Speculatively, the presence of childhood adversity might impact the typical developmental trajectory of perceptual and causal reasoning systems, meaning that greater numbers of PLEs largely reflect developmental delay in these systems rather than dysregulation of striatal dopamine at this age. Nevertheless, accumulation of pre-existing and environmental risk factors, particularly those that have a broader impact on development ${ }^{56}$ will make later hyper-responsiveness of the striatal dopamine system more likely. This interactive developmental model might account for the contrasting trajectories of PLEs from childhood to adolescence where the majority of children show resolving or attenuated PLEs and only a small high-risk minority show persistent or intermittent PLEs. ${ }^{57}$

However, this study presents several limitations that warrant caution when interpreting its results. One limitation is the extent to which blood-oxygen-level-dependent (BOLD) signal in the ventral striatum allows accurate localization of dysregulated subcortical dopamine. Metaanalytic evidence from PET studies suggests that it may be the dorsal rather than ventral striatum where dopamine dysregulation may be most apparent in adult psychosis. ${ }^{58}$ The area of interest used in this study was the NAcc, based in the ventral striatum. Nevertheless, activation in this area during the MID task is reliably associated with psychosis across meta-analysis of multiple studies. ${ }^{21}$ Therefore, it is likely that BOLD signal response reliably reflects altered dopamine-mediated reward processing, but it may not accurately localize it. Indeed, a prior multimodal PET-fMRI study of the MID task ${ }^{24}$ reported that reward anticipation was reliably associated with BOLD signal in the NAcc with PET imaging showed it was associated with dopamine transporter availability in the midbrain. Consequently, we presume it is unlikely that there would be marked dysregulation to functionally adjacent areas in the dopamine system that would not result in BOLD detectable NAcc activation during the MID task, meaning it likely remains a useful measure of altered dopamine-mediated reward processing. However, given these validation studies were conducted in adults, we also note the limitations of generalizing this assumption to 9-10-year-old children. Given that PET studies are typically used to cross-validate the role a dopamine in regional fMRI activation and are not routinely conducted on children except for clinical reasons, this may be difficult to directly test, but remains a possibility.

Although the ABCD endeavored to obtain a nationally representative sample, children from higher income families were over-represented. We attempted to address this by including family and site as random effects as suggested by Heeringa and Berglund, ${ }^{59}$ although it is possible that this did not fully eliminate sampling biases. PLEs were measured with the Prodromal Questionnaire-Brief Child Version. This is a self-report questionnaire and although has been well validated in this sample ${ }^{32}$ may not have had the same sensitivity as structured interview assessments.

We note that the sole positive statistical association reported in this study was between reward anticipation and distressing PLEs in children grouped by having any psychiatric diagnosis. We also note that this finding was small and seemingly very selective-it was not present for reward outcome and was not associated with nondistressing PLEs in the same analysis. However, it is possible that this group represents a subgroup where the earliest effects of dopamine system dysregulation may be found, potentially related more broadly to psychopathology, and this may be worth noting as a hypothesis for future investigation. 
In conclusion, we found no reliable evidence that the presence of PLEs in a large and well-powered sample predicted dysregulated reward processing. As the ABCD study is ongoing cohort study, future research should focus on exploring the timing of PLE-related differences in reward processing arise during development.

\section{Supplementary Material}

Supplementary data are available at Schizophrenia Bulletin Open online.

\section{Funding}

JHS: MRC doctoral studentship. HI: EU Horizon 2020 under a Marie Sklodowska-Curie grant (747429); National Institute of Allergy and Infectious Diseases. EB: NIHR UK (NIHR200756); Mental Health Research UK; ESRC; BMA Margaret Temple Fellowship; MRC New Investigator and Centenary Awards (G0901310 and G1100583). NIHR Biomedical Research Centre at UCL Hospitals and UCL. SJB: Wellcome (grant number WT107496/Z/15/Z); Jacobs Foundation; Wellspring Foundation; University of Cambridge. LM: MRC Clinician Scientist Fellowship (MR/S006613/1). VB is funded by the UKRI Global Challenges Research Fund.

\section{Acknowledgments}

Data used in the preparation of this article were obtained from the Adolescent Brain Cognitive Development ${ }^{\mathrm{SM}}$ (ABCD) Study (https://abcdstudy.org), held in the NIMH Data Archive (NDA). This is a multisite, longitudinal study designed to recruit more than 10000 children age 9-10 and follow them over 10 years into early adulthood. The ABCD Study is supported by the National Institutes of Health and additional federal partners under award numbers U01DA041022, U01DA041028, U01DA041048, U01DA041089, U01DA041106, U01DA041117, U01DA041120, U01DA041134, U01DA041148, U01DA041156, U01DA041174, U24DA041123, U24DA041147, U01DA041093, and U01DA041025. A full list of supporters is available at https://abcdstudy.org/federal-partners.html. A listing of participating sites and a complete listing of the study investigators can be found at https://abcdstudy. org/Consortium_Members.pdf. ABCD consortium investigators designed and implemented the study and/ or provided data but did not necessarily participate in analysis or writing of this report. This manuscript reflects the views of the authors and may not reflect the opinions or views of the NIH or ABCD consortium investigators. The $\mathrm{ABCD}$ data repository grows and changes over time. The ABCD data used in this report came from doi:10.15154/1521353. DOIs can be found at https://doi.org.

\section{References}

1. Linscott RJ, van Os J. An updated and conservative systematic review and meta-analysis of epidemiological evidence on psychotic experiences in children and adults: on the pathway from proneness to persistence to dimensional expression across mental disorders. Psychol Med. 2013;43(6):1133-1149.

2. Kelleher I, Connor D, Clarke MC, Devlin N, Harley M, Cannon M. Prevalence of psychotic symptoms in childhood and adolescence: a systematic review and metaanalysis of population-based studies. Psychol Med. 2012;42(9):1857-1863.

3. Fisher HL, Caspi A, Poulton R, et al. Specificity of childhood psychotic symptoms for predicting schizophrenia by 38 years of age: a birth cohort study. Psychol Med. 2013;43(10):2077-2086.

4. Healy C, Brannigan R, Dooley N, et al. Childhood and adolescent psychotic experiences and risk of mental disorder: a systematic review and meta-analysis. Psychol Med. 2019;49(10):1589-1599.

5. Poulton R, Caspi A, Moffitt TE, Cannon M, Murray R, Harrington H. Children's self-reported psychotic symptoms and adult schizophreniform disorder: a 15-year longitudinal study. Arch Gen Psychiatry. 2000;57(11):1053-1058.

6. Davies J, Sullivan S, Zammit S. Adverse life outcomes associated with adolescent psychotic experiences and depressive symptoms. Soc Psychiatry Psychiatr Epidemiol. 2018;53(5):497-507.

7. Trotta A, Arseneault L, Caspi A, et al. Mental health and functional outcomes in young adulthood of children with psychotic symptoms: a longitudinal cohort study. Schizophr Bull. 2020;46(2):261-271.

8. Macmanus D, Laurens KR, Walker EF, Brasfield JL, Riaz M, Hodgins S. Movement abnormalities and psychoticlike experiences in childhood: markers of developing schizophrenia? Psychol Med. 2012;42(1):99-109.

9. Howes OD, Kapur S. The dopamine hypothesis of schizophrenia: version III - the final common pathway. Schizophr Bull. 2009;35(3):549-562.

10. Murray RM, Bhavsar V, Tripoli G, Howes O. 30 years on: how the neurodevelopmental hypothesis of schizophrenia morphed into the developmental risk factor model of psychosis. Schizophr Bull. 2017;43(6):1190-1196.

11. Howes OD, Hird EJ, Adams RA, Corlett PR, McGuire P. Aberrant salience, information processing, and dopaminergic signaling in people at clinical high risk for psychosis. Biol Psychiatry. 2020;88(4):304-314.

12. Papanastasiou E, Mouchlianitis E, Joyce DW, et al.; IMAGEN Consortium. Examination of the neural basis of psychoticlike experiences in adolescence during reward processing. JAMA Psychiatry. 2018;75(10):1043-1051.

13. Rubio JM, Sanjuán J, Flórez-Salamanca L, Cuesta MJ. Examining the course of hallucinatory experiences in children and adolescents: a systematic review. Schizophr Res. 2012;138(2-3):248-254.

14. van Os J, Linscott RJ, Myin-Germeys I, Delespaul P, Krabbendam L. A systematic review and meta-analysis of the psychosis continuum: evidence for a psychosis pronenesspersistence-impairment model of psychotic disorder. Psychol Med. 2009;39(2):179-195.

15. Calkins ME, Moore TM, Satterthwaite TD, et al. Persistence of psychosis spectrum symptoms in the Philadelphia Neurodevelopmental Cohort: a prospective two-year follow-up. World Psychiatry. 2017;16(1):62-76. 
16. Downs JM, Cullen AE, Barragan M, Laurens KR. Persisting psychotic-like experiences are associated with both externalising and internalising psychopathology in a longitudinal general population child cohort. Schizophr Res. 2013;144(1-3):99-104.

17. Sullivan SA, Kounali D, Cannon M, et al. A populationbased cohort study examining the incidence and impact of psychotic experiences from childhood to adulthood, and prediction of psychotic disorder. Am J Psychiatry. 2020;177(4):308-317.

18. Schimmelmann BG, Michel C, Martz-Irngartinger A, Linder C, Schultze-Lutter F. Age matters in the prevalence and clinical significance of ultra-high-risk for psychosis symptoms and criteria in the general population: findings from the BEAR and BEARS-kid studies. World Psychiatry. 2015;14(2):189-197.

19. Schultz W. Getting formal with dopamine and reward. Neuron. 2002;36(2):241-263.

20. Knutson B, Westdorp A, Kaiser E, Hommer D. FMRI visualization of brain activity during a monetary incentive delay task. Neuroimage. 2000;12(1):20-27.

21. Radua J, Schmidt A, Borgwardt S, et al. Ventral striatal activation during reward processing in psychosis: a neurofunctional meta-analysis. JAMA Psychiatry. 2015;72(12):1243-1251.

22. Nielsen MØ, Rostrup E, Wulff S, et al. Alterations of the brain reward system in antipsychotic naïve schizophrenia patients. Biol Psychiatry. 2012;71(10):898-905.

23. Wotruba D, Heekeren K, Michels L, et al. Symptom dimensions are associated with reward processing in unmedicated persons at risk for psychosis. Front Behav Neurosci. $2014 ; 8: 382$.

24. Dubol M, Trichard C, Leroy C, et al. Dopamine transporter and reward anticipation in a dimensional perspective: a multimodal brain imaging study. Neuropsychopharmacology. 2018;43(4):820-827.

25. Dougherty LR, Schwartz KTG, Kryza-Lacombe M, Weisberg J, Spechler PA, Wiggins JL. Preschool- and schoolage irritability predict reward-related brain function. $J \mathrm{Am}$ Acad Child Adolesc Psychiatry. 2018;57(6):407-417.e2.

26. Turner BO, Paul EJ, Miller MB, Barbey AK. Small sample sizes reduce the replicability of task-based fMRI studies. Commun Biol. 2018;1:62.

27. Herting MM, Gautam P, Chen Z, Mezher A, Vetter NC. Test-retest reliability of longitudinal task-based fMRI: implications for developmental studies. Dev Cogn Neurosci. 2018;33:17-26.

28. Garavan $\mathrm{H}$, Bartsch $\mathrm{H}$, Conway $\mathrm{K}$, et al. Recruiting the ABCD sample: design considerations and procedures. Dev Cogn Neurosci. 2018;32:16-22.

29. Volkow ND, Koob GF, Croyle RT, et al. The conception of the ABCD study: from substance use to a broad NIH collaboration. Dev Cogn Neurosci. 2018;32:4-7.

30. Clark DB, Fisher CB, Bookheimer S, et al. Biomedical ethics and clinical oversight in multisite observational neuroimaging studies with children and adolescents: the ABCD experience. Dev Cogn Neurosci. 2018;32:143-154.

31. Loewy RL, Pearson R, Vinogradov S, Bearden CE, Cannon TD. Psychosis risk screening with the Prodromal Questionnaire-brief version (PQ-B). Schizophr Res. 2011;129(1):42-46.

32. Karcher NR, Barch DM, Avenevoli S, et al. Assessment of the prodromal questionnaire-brief child version for measurement of self-reported psychotic-like experiences in childhood. JAMA Psychiatry. 2018;75(8):853-861.

33. Townsend L, Kobak K, Kearney C, et al. Development of three web-based computerized versions of the Kiddie Schedule for affective disorders and schizophrenia child psychiatric diagnostic interview: preliminary validity data. $J \mathrm{Am}$ Acad Child Adolesc Psychiatry. 2020;59(2):309-325.

34. Petersen AC, Crockett L, Richards M, Boxer A. A self-report measure of pubertal status: reliability, validity, and initial norms. J Youth Adolesc. 1988;17(2):117-133.

35. Casey BJ, Cannonier T, Conley MI, et al.; ABCD Imaging Acquisition Workgroup. The Adolescent Brain Cognitive Development (ABCD) study: imaging acquisition across 21 sites. Dev Cogn Neurosci. 2018;32:43-54.

36. Cao Z, Bennett M, Orr C, et al.; IMAGEN Consortium. Mapping adolescent reward anticipation, receipt, and prediction error during the monetary incentive delay task. Hum Brain Mapp. 2019;40(1):262-283.

37. Chaarani B, Hahn S, Allgaier N, et al.; ABCD Consortium. Baseline brain function in the preadolescents of the ABCD Study. Nat Neurosci. 2021;24(8):1176-1186.

38. Hagler DJ Jr, Hatton S, Cornejo MD, et al. Image processing and analysis methods for the Adolescent Brain Cognitive Development Study. Neuroimage. 2019;202:116091.

39. Fischl B. FreeSurfer. Neuroimage. 2012;62(2):774-781.

40. Koller M. robustlmm: an R package for robust estimation of linear mixed-effects models. J Stat Softw. 2016;75(1):1-24.

41. Jongsma HE, Gayer-Anderson C, Tarricone I, et al. Social disadvantage, linguistic distance, ethnic minority status and first-episode psychosis: results from the EU-GEI case-control study. Psychol Med. Published online 2021;51(9):1536-1548.

42. Morgan C, Fisher H, Hutchinson G, et al. Ethnicity, social disadvantage and psychotic-like experiences in a healthy population based sample. Acta Psychiatr Scand. 2009;119(3):226-235.

43. Murphy D, Vallières F, Murphy J, McElroy E, Hyland P. Risk factors associated with general and specific dimensions of psychosis in a nationally representative sample of adults from the United States. Psychosis. 2020;12(4):303-313.

44. Keren H, O'Callaghan G, Vidal-Ribas P, et al. Reward processing in depression: a conceptual and meta-analytic review across fMRI and EEG studies. Am J Psychiatry. 2018;175(11):1111-1120.

45. Ladouceur CD, Kerestes R, Schlund MW, Shirtcliff EA, Lee Y, Dahl RE. Neural systems underlying reward cue processing in early adolescence: the role of puberty and pubertal hormones. Psychoneuroendocrinology. 2019;102:281-291.

46. Buuren $\mathrm{S}$ van, Groothuis-Oudshoorn K. mice: multivariate imputation by chained equations in R. J Stat Softw. 2011;45(3):1-67.

47. Wickham $H$. Reshaping data with the reshape package. $J$ Stat Softw. 2007;21(12):1-20.

48. Kapur S. Psychosis as a state of aberrant salience: a framework linking biology, phenomenology, and pharmacology in schizophrenia. AJP. 2003;160(1):13-23.

49. Juckel G, Schlagenhauf F, Koslowski M, et al. Dysfunction of ventral striatal reward prediction in schizophrenia. Neuroimage. 2006;29(2):409-416.

50. Kirschner M, Hager OM, Muff L, et al. Ventral striatal dysfunction and symptom expression in individuals with schizotypal personality traits and early psychosis. Schizophr Bull. 2018;44(1):147-157. 
51. Reichenberg A, Caspi A, Harrington $\mathrm{H}$, et al. Static and dynamic cognitive deficits in childhood preceding adult schizophrenia: a 30-year study. Am J Psychiatry. 2010;167(2):160-169.

52. Trotta A, Murray RM, Fisher HL. The impact of childhood adversity on the persistence of psychotic symptoms: a systematic review and meta-analysis. Psychol Med. 2015;45(12):2481-2498.

53. Moore DR. Listening difficulties in children: bottom-up and top-down contributions. $J$ Commun Disord. 2012;45(6):411-418.

54. Peters JC, Kemner C. Facial expressions perceived by the adolescent brain: towards the proficient use of low spatial frequency information. Biol Psychol. 2017;129:1-7.

55. Muentener P, Bonawitz E. The Development of Causal Reasoning. OSF Preprints; 2018.
56. Dominguez MD, Saka MC, can Saka M, Lieb R, Wittchen HU, van Os J. Early expression of negative/disorganized symptoms predicting psychotic experiences and subsequent clinical psychosis: a 10-year study. Am J Psychiatry. 2010;167(9):1075-1082.

57. Thapar A, Heron J, Jones RB, Owen MJ, Lewis G, Zammit S. Trajectories of change in self-reported psychotic-like experiences in childhood and adolescence. Schizophr Res. 2012; 140(1-3):104-109.

58. McCutcheon R, Beck K, Jauhar S, Howes OD. Defining the locus of dopaminergic dysfunction in schizophrenia: a metaanalysis and test of the mesolimbic hypothesis. Schizophr Bull. 2018;44(6):1301-1311.

59. Heeringa SG, Berglund PA. A guide for population-based analysis of the adolescent brain cognitive development (ABCD) study baseline data. Neuroscience. 2020. 